\title{
Diversidade e potencial de infectividade de fungos micorrízicos arbusculares em área de caatinga, na Região de Xingó, Estado de Alagoas, Brasil ${ }^{1}$
}

\author{
RENATA G. SOUZA ${ }^{2}$, LEONOR C. MAIA ${ }^{2,5}$, MARGARETH F. SALES $^{3}$ e SANDRA F.B. TRUFEM ${ }^{4}$
}

(recebido: 17 de abril de 2002; aceito: 25 de outubro de 2002)

\begin{abstract}
Diversity and infectivity potential of arbuscular mycorrhizal fungi in an area of "caatinga" in the Xingó Region, State of Alagoas, Brazil). The region occupies $2800 \mathrm{~km}^{2}$, in the States of Pernambuco, Alagoas, Sergipe and Bahia, and constitutes a preserved part of the Northeastern semi-arid ecosystem. Evaluation of the diversity and density of propagules of arbuscular mycorrhizal fungi (AMF) in the soil, as well as of the mycorrhizal colonization in plants of the area was carried out by sampling of soil and roots during the dry (August/2000) and rainy (March/2001) seasons, in two subareas (Piranhas and Olho d'Água do Casado) in Alagoas. More than 95\% of the plants, among the 71 examined, formed arbuscular mycorrhiza (5-80\% colonization). Among these 30 phanerogamic species, belonging to 14 families, only Pilosocereus sp. was not colonized.

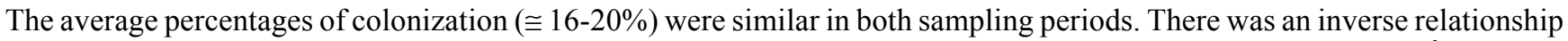
between number of spores and the most probable number (MPN) of infective propagules in the area of Olho d'Água, with lower density of spores ( $<2$ spores. $\mathrm{g}^{-1}$ of soil) and higher MPN of propagules (4.7 and 11.6 spores. $^{-1}$ of soil), in the rainy and dry periods. Number of spores and MPN of propagules were similar in the Piranhas area during the rainy season; in the dry season the number of spores was 1.5 times higher than the MPN. Twenty-four taxa of AMF, mostly of Acaulosporaceae and Glomaceae, were identified. The AMF are well represented, forming mycorrhizal association with most "caatinga" plants, despite the severe climatic limitations of the region.
\end{abstract}

Key words - Arbuscular mycorrhiza, caatinga, diversity, infectivity potential, trap culture

RESUMO - (Diversidade e potencial de infectividade de fungos micorrízicos arbusculares em área de Caatinga na Região de Xingó, Estado de Alagoas, Brasil). A região de Xingó ocupa 2.800 km², em Pernambuco, Alagoas, Sergipe e Bahia, constituindo parte ainda conservada do semi-árido nordestino. Para avaliação da diversidade e da densidade de propágulos de FMA no solo e da colonização micorrízica em plantas da área, foram realizadas coletas de solo e raízes nas estações seca (agosto/2000) e chuvosa (março/2001), em duas subáreas de Piranhas e Olho d’Água do Casado, em Alagoas. Mais de 95\% das plantas, dentre as 71 examinadas, apresentaram micorriza arbuscular (5\% a 80\%). Das 30 espécies de fanerógamas, correspondentes a 14 famílias, apenas Pilosocereus sp. não estava associado com FMA. Os percentuais médios de colonização (@16 a $20 \%$ ) foram semelhantes nos dois períodos. Houve relação inversa entre o número de esporos e o número mais provável (NMP) de propágulos infectivos em Olho d'Água do Casado, com menor densidade de esporos ( $<2$ esporos.g-1 de solo) e maior NMP de propágulos (4,7 e 11,6 esporos. $\mathrm{g}^{-1}$ de solo), nos períodos chuvoso e seco, respectivamente. Em Piranhas o número de esporos e o NMP de propágulos foram similares no período chuvoso, enquanto no período seco houve 1,5 vezes mais esporos do que propágulos infectivos. Foram identificados 24 táxons de FMA, com maior representatividade de Acaulosporaceae e Glomaceae. Os FMA estão bem representados, formando associação com a maioria das espécies de caatinga, apesar das limitações climáticas da região.

Palavras-chave - Caatinga, cultura armadilha, diversidade, micorriza arbuscular, potencial de infectividade

\section{Introdução}

Como simbiontes obrigatórios os fungos micorrízicos arbusculares (FMA) trazem benefícios à comunidade

1. Parte da dissertação de mestrado de R.G.Souza, vinculada ao Programa de Pós-Graduação em Biologia de Fungos da Universidade Federal de Pernambuco.

2. Universidade Federal de Pernambuco, Centro de Ciências Biológicas, Depto. de Micologia, Cidade Universitária, 50670-420 Recife, PE, Brasil

3. Universidade Federal Rural de Pernambuco, Depto. de Biologia/ Área Botânica, Dois Irmãos, 52171-900 Recife, PE

4. Instituto de Botânica, Caixa Postal 4005, 01061-970 São Paulo, SP, Brasil.

5. Autor para correspondência: leonorcmaia@hotmail.com vegetal e ao ambiente, fornecendo nutrientes e água às plantas, assim como favorecendo a retenção de umidade, a agregação e a estabilidade dos solos (Sylvia 1992, Augé et al. 2001).

As micorrizas arbusculares são importantes não só por promover a interface entre a planta e o ambiente físico, mas também com o ambiente biológico, conferindo às plantas maior resistência e tolerância a estresses bióticos e abióticos, além de influenciar a diversidade vegetal (Miller \& Kling 2000). Entre os diversos tipos de micorrizas, as arbusculares, formadas por fungos Glomales (Zygomycetes), são de particular importância nos trópicos, onde estão melhor 
distribuídas e ocorrem com maior freqüência (Smith \& Read 1997).

A região semi-árida do Brasil ocupa cerca de $800.000 \mathrm{~km}^{2}$, totalizando $11 \%$ do território nacional (Drumond et al. 2000). Nesta região prevalece o clima semi-árido, caracterizado pelo elevado potencial de evapotranspiração $\left(2.000 \mathrm{~mm} . \mathrm{ano}^{-1}\right)$, precipitação média anual de $700 \mathrm{~mm}$ (mínima de 300 e máxima de $1.000 \mathrm{~mm}$ ) concentradas em três a cinco meses do ano e temperatura média anual de 23 a $27{ }^{\circ} \mathrm{C}$ (Sampaio 1995). A vegetação típica é a caatinga, bioma único no mundo, caracterizado pela formação de floresta seca composta de vegetação xerófila de porte arbóreo, arbustivo e herbáceo, com ampla variação de fisionomia e flora e elevada diversidade de espécies, predominando representantes de Caesalpinaceae, Mimosaceae, Euphorbiaceae, Fabaceae e Cactaceae (Drumond et al. 2000).

A caatinga encontra-se hoje em acentuado processo de desertificação, ocasionado, principalmente, pelo desmatamento e uso inadequado dos recursos naturais (Drumond et al. 2000). A desertificação resulta na redução de produção vegetal, acarretando mudanças nas interações que ocorrem no solo, com a conseqüente e muitas vezes irreversível perda da biodiversidade (Skujins \& Allen 1986). Nas regiões áridas e semiáridas a baixa fertilidade dos solos gera elevada dependência das plantas pelos FMA, que minimizam os estresses hídricos e a deficiência de nutrientes (Tarafdar \& Praveen-Kumar 1996), sendo importante, portanto, conhecer a diversidade de FMA nessas áreas.

A diversidade, a densidade e o potencial de infectividade dos propágulos de FMA no solo estão relacionados indiretamente com as condições ecológicas de cada ecossistema (Maia \& Trufem 1990) e diretamente com a fisiologia do fungo (Morton 1993), estando a colonização micorrízica ligada ao genótipo da planta e do fungo, assim como ao ambiente.

Considerando o relevante papel desempenhado pelos fungos micorrízicos arbusculares, procurou-se neste estudo conhecer a diversidade, similaridade de espécies e densidade de propágulos de FMA, bem como a colonização micorrízica de plantas em área de caatinga.

\section{Material e métodos}

Áreas de estudo - Após a construção da hidroelétrica de Xingó, em 1994, a área circunvizinha passou a ser objeto de estudo, entre os quais se inclui o da biodiversidade animal, vegetal e de fungos. Neste contexto, foram selecionadas quatro subáreas, em duas fazendas: Baixa da Légua (Latitude 962'44" S e Longitude 37 $75^{\prime} 69^{\prime}$ ' W) e Capelinha (Latitude $9^{\circ} 50^{\prime} 83^{\prime}$ ' S e Longitude $37^{\circ} 83$ ' $22^{\prime}$ ' W), situadas respectivamente nos municípios de Piranhas e Olho d'Água do Casado, Estado de Alagoas.

O clima local é característico do semi-árido, com precipitação e temperaturas médias anuais de $500 \mathrm{~mm}$ e 24 a $26{ }^{\circ} \mathrm{C}$, respectivamente. De acordo com os dados de precipitação média mensal dos dois municípios, a média de precipitação no período de março/2000 a março/2001 foi 427,2 mm no município de Piranhas e 596,3 $\mathrm{mm}$ em Olho d'Água do Casado. As áreas experimentais do município de Piranhas estão situadas em área de domínio de Planossolo, caracterizado por solos de textura franco-arenosa, pouco profundos, de textura média no horizonte $\mathrm{A}$ e argilosa no horizonte $\mathrm{B}$, apresentando drenagem imperfeita. As do município de Olho d'Água do Casado estão situadas em área de domínio de Regossolo, caracterizado por solos de textura franco-arenosa, de profundidade média, excessivamente drenados, apresentando minerais primários de fácil intemperização (Jacomine et al. 1975).

A área é coberta por vegetação caducifólia espinhosa (caatinga), arbustivo-arbórea em geral aberta, entremeada por poucos indivíduos arbóreos, emergentes na paisagem geral. O estrato herbáceo é quase ausente no período seco. Na flora destacam-se espécies de Caesalpinia, Mimosa, Anadenanthera, Bauhinia e Pithecellobium, entre outros. Compondo o estrato arbustivo dominam Euphorbiaceae (Croton, Cnidoscolus e Jatropha); também são freqüentes indivíduos arbóreos de Zizyphus joazeiro Mart. e Spondias tuberosa Arr. Cam., e sobre os afloramentos rochosos são comuns espécies de Cactaceae e Bromeliaceae. De acordo com Sampaio (1995), as plantas de maior densidade na caatinga são, de porte arbóreo, Caesalpinia pyramidalis Tul. e Aspidosperma pyrifolium Mart.; de porte arbustivo, espécies de Croton e Mimosa e, do baixo estrato, Bromelia laciniosa Mart. ex Schultes f.

Coletas - Foram realizadas no período seco (agosto/2000) e chuvoso (março/2001), sendo escolhidas duas subáreas em Piranhas (PA e PB) e duas em Olho d'Água do Casado (OA e OB), com vegetação típica de caatinga. Para cada subárea, com dimensão de $1.000 \mathrm{~m}^{2}$, foram definidos nove pontos aleatórios, traçados em zig-zag. Em cada ponto foi selecionada uma planta e efetuada coleta de aproximadamente $1 \mathrm{~kg}$ de solo da rizosfera até a profundidade de $20 \mathrm{~cm}$. As amostras foram acondicionadas em sacos plásticos para posterior determinação da densidade de esporos, número de propágulos infectivos e identificação das espécies de FMA. Das plantas foram coletados: fragmentos de raízes, inflorescências e parte vegetativa para identificação das espécies. Exsicatas foram depositadas no Herbário Professor Vasconcelos Sobrinho, da Universidade Federal Rural de Pernambuco (UFRPE).

Análises físicas e químicas do solo - De cada amostra composta, constituída por solo dos nove pontos de coleta em 
cada subárea, foram retiradas amostras e encaminhadas para análise química (Empresa Pernambucana de Pesquisa Agropecuária - IPA) e física (Laboratório de Física do Solo - UFRPE) (tabela 1). Os solos apresentam as seguintes características, segundo Tomé Júnior (1997): acidez da solução aquosa de média $(5,2 ; 5,6$ e 5,8$)$ a fraca $(6,2)$; teores de fósforo baixos para as subáreas de Piranhas $\left(<7 \mathrm{mg} \cdot \mathrm{dm}^{-3}\right)$ e altos para as de Olho d'Água do Casado ( $\left.>40 \mathrm{mg} . \mathrm{dm}^{-3}\right)$; cálcio e potássio variando de médio a alto; magnésio com alto teor, variando de 1,3 a $2,75 \mathrm{cmol}_{\mathrm{c}} \cdot \mathrm{dm}^{-3}$.

Avaliação da colonização micorrízica - As raízes foram lavadas, diafanizadas com $\mathrm{KOH}(10 \%)$ e $\mathrm{H}_{2} \mathrm{O}_{2}$, acidificadas com $\mathrm{HCl}(1 \%)$ e coradas com azul de Tripano em lactoglicerol (0,05\%) (Phillips \& Hayman 1970). A colonização foi avaliada pelo método de interseção dos quadrantes (Giovannetti \& Mosse 1980), sendo realizadas três observações por sistema radicular e o percentual de colonização de cada amostra obtido pela média aritmética. Determinação da densidade de esporos - As amostras de solo foram peneiradas (malha de $5 \mathrm{~mm}$ ) e homogeneizadas, sendo retiradas duas subamostras de $50 \mathrm{~g}$ para extração dos esporos de FMA pelo método de peneiramento em via úmida (Gerdemann \& Nicolson 1963), seguido de centrifugações em água e sacarose (Jenkins 1964, modificado pelo uso de sacarose $40 \%$ e centrifugação a $2.500 \mathrm{rpm}$ ) e contagem direta em placas canaletadas. A densidade de esporos em cada área foi obtida pela média do número de esporos de cada ponto de coleta.

Determinação do Número Mais Provável (NMP) de propágulos infectivos - Para avaliação do NMP de propágulos de FMA foi utilizada a técnica descrita em Sieverding (1991). Para cada período (seco e chuvoso) foram montados quatro bioensaios. As amostras teste (350 g de solo) foram obtidas a partir da homogeneização de subamostras dos nove pontos de coleta de cada subárea. Além da coleta de solo das amostras teste, foi realizada coleta de solo em cada uma das áreas, para ser usado como diluente e na composição dos potes do bioensaio. Este solo foi peneirado (malha de $5 \mathrm{~mm}$ ) e autoclavado por $1 \mathrm{~h}$ a $120{ }^{\circ} \mathrm{C}$ (1 atm) por dois dias consecutivos e seco em estufa a $105^{\circ} \mathrm{C}$. Foi utilizado o fator quatro como base de diluição 3:1 (diluente : amostra), com cinco repetições para cada nível, totalizando nove níveis, de $4^{0}$ a $4^{-8}$. Para cada subárea foram preparados 45 potes. No total, os bioensaios incluíram 360 potes, considerando: 2 momentos de coleta x 4 subáreas x 9 níveis de diluição e 5 repetições. Cada pote foi preenchido com $150 \mathrm{~g}$ do substrato $\mathrm{B}$ (solo autoclavado) $+50 \mathrm{~g}$ do substrato A (solo autoclavado + solo teste $)+50 \mathrm{~g}$ do substrato $\mathrm{B}+20 \mathrm{~g}$ de areia lavada e autoclavada. Vinte sementes de Panicum miliaceum L., desinfestadas com $\mathrm{NaOCl}$ a $10 \%$ foram colocadas em cada pote. Após 15 dias da germinação, foi realizado o desbaste mantendo-se 10 plantas por pote, que permaneceram em telado durante dois meses. A temperatura e a umidade relativa do ar foram medidas diariamente, variando de $23{ }^{\circ} \mathrm{C}$ a $32{ }^{\circ} \mathrm{C}$ e de $50 \%$ a $81 \%$ respectivamente (termohigrômetro TFA, Alemanha). No final do período as plantas foram colhidas e as raízes separadas, lavadas e mantidas em álcool a 50\%, para serem depois diafanizadas e coradas como mencionado. O solo retirado dos potes (níveis $0,1,2,3$, e 4) foi homogeneizado e acondicionado em sacos plásticos, para obtenção do segundo ciclo de multiplicação dos esporos, com o objetivo de identificar posteriormente as espécies de FMA.

Para avaliação do potencial de infectividade de FMA no solo, foram atribuídos os sinais $(+)$ para presença e $(-)$ para ausência de estruturas típicas de FMA, quando da observação das raízes, em estereomicroscópio. Para cálculo do NMP de propágulos infectivos foi utilizada a fórmula: $\log \Omega=\mathrm{x} \log \mathrm{a}-\mathrm{K}$ onde:

$\Omega=$ número de propágulos infectivos;

$\mathrm{x}=$ número total de potes infectados / número de repetições por diluição;

$\mathrm{a}=$ fator de diluição;

$\mathrm{K}=$ constante encontrada na tabela VIII de Fisher \& Yates (1970), determinada pelos valores de $\mathrm{x}$ e $\mathrm{y}$, onde $\mathrm{y}=\mathrm{s}-\mathrm{x}$, sendo s o número do nível de diluição.

Identificação das espécies de FMA - Para identificação das espécies foram retiradas amostras do solo nativo, do solo usado nos bioensaios do NMP e ainda do solo proveniente de culturas armadilhas, as quais foram preparadas com os solos de cada subárea, usando como hospedeiro P. miliaceum,

Tabela 1. Características químicas e físicas de solos das áreas experimentais, nos municípios de Piranhas e Olho d'Água do Casado, Alagoas. (PA e PB = áreas de Piranhas, OA e OB = áreas de Olho d'Água do Casado).

Table 1. Chemical and physical characteristics of soils of the experimental sites, at the municipalities of Piranhas and Olho d'Água do Casado, state of Alagoas. (PA and PB = Piranhas' areas, OA and OB = Olho d'Água do Casado's areas).

\begin{tabular}{|c|c|c|c|c|c|c|c|c|c|}
\hline \multirow[t]{2}{*}{ SUBÁREA } & $\mathrm{pH}$ & $\mathrm{P}$ & K & $\mathrm{Al}$ & $\mathrm{Ca}$ & \multirow[t]{2}{*}{$\mathrm{Mg}$} & AREIA & ARGILA & SILTE \\
\hline & $\mathrm{H}_{2} \mathrm{O}$ & $\mathrm{mg} \mathrm{dm}^{-3}$ & \multicolumn{3}{|c|}{$\mathrm{cmol}_{\mathrm{c}} \mathrm{dm}^{-3}$} & & \multicolumn{3}{|c|}{$\%$} \\
\hline PA & 5,80 & 5 & 0,25 & 0,00 & 4,70 & 2,75 & 62,5 & 15,5 & 22,0 \\
\hline PB & 5,60 & 6 & 0,21 & 0,05 & 2,85 & 1,30 & 74,5 & 10,5 & 15,0 \\
\hline OA & 5,20 & $>40$ & 0,24 & 0,10 & 4,00 & 1,35 & 77,5 & 10,5 & 12,0 \\
\hline $\mathrm{OB}$ & 6,20 & $>40$ & 0,34 & 0,00 & 6,40 & 2,40 & 72,5 & 12,5 & 15,0 \\
\hline
\end{tabular}


e mantidas em telado por três meses. Os esporos foram extraídos conforme descrito, montados em lâminas com PVLG (álcool-polivinílico em lactoglicerol) ou com reagente de Melzer + PVLG (1:1) e observados ao microscópio. Para identificação foram consultados o Manual para Identificação de FMA (Schenck \& Pérez 1990), a home page http://invam.caf.wvu.edu e publicações com a descrição de novas espécies.

Análise estatística - Para análise, os números de esporos foram transformados em $\log (\mathrm{x}+1)$ e os dados do NMP em $\log (\mathrm{x})$, para comparação das médias pelo teste de Tukey a 5\% de probabilidade, usando o programa Statistica (1995). A similaridade de espécies de FMA entre as áreas foi avaliada pelo Índice de Sörensen (Brower \& Zar 1984).

\section{Resultados}

Foram identificados 24 táxons de FMA; maior diversidade ocorreu no período seco, com as subáreas do município de Piranhas apresentando maior riqueza de espécies (tabela 2). O índice de similaridade das espécies de FMA entre os dois municípios foi de 54,5\%.

A densidade de esporos de FMA na rizosfera das plantas variou de 0,34 a 8,6 esporos. $\mathrm{g}^{-1}$ de solo (tabela 3). Em Olho d'Água do Casado, apenas uma amostra apresentou densidade igual ou superior a 5 esporos. $\mathrm{g}^{-1}$ de solo, no período seco; no chuvoso a densidade foi sempre menor que 4,1 esporos. $\mathrm{g}^{-1}$ de solo. Em Piranhas, a maior densidade de esporos foi encontrada na rizosfera de imbira $\left(8,5\right.$ esporos. $\mathrm{g}^{-1}$ de solo), no período seco. Não foi observada relação entre as espécies de plantas, percentuais de colonização e a densidade de esporos na rizosfera.

No município de Piranhas, a densidade de esporos de FMA em cada área foi significativamente maior no período seco do que no chuvoso; por outro lado, não houve diferença entre as subáreas (tabela 4). Em Olho

Tabela 2. Espécies de FMA isoladas do solo das áreas experimentais nos municípios de Piranhas e Olho d'Água do Casado, Alagoas, no período seco (PS) e chuvoso (PC).

Table 2. FMA species isolated from soil of the experimental sites at the municipalities of Piranhas and Olho d'Água do Casado, state of Alagoas, in the dry (PS) and wet (PC) seasons

\begin{tabular}{|c|c|c|c|c|}
\hline \multirow[t]{2}{*}{ FMA } & \multicolumn{2}{|c|}{ Piranhas } & \multicolumn{2}{|c|}{ Olho d'Água } \\
\hline & PS & $\mathrm{PC}$ & PS & $\mathrm{PC}$ \\
\hline Acaulospora denticulata Sieverding \& Toro & $\mathrm{x}$ & $\mathrm{x}$ & & $\mathrm{x}$ \\
\hline Acaulospora excavata Ingleby, Walker \& Mason & $\mathrm{x}$ & $\mathrm{x}$ & $\mathrm{x}$ & $\mathrm{x}$ \\
\hline Acaulospora lacunosa Morton & & $\mathrm{x}$ & & \\
\hline Acaulospora longula Spain \& Schenck & & $\mathrm{x}$ & & \\
\hline Acaulospora rehmii Sieverding \& Toro & $\mathrm{x}$ & & & \\
\hline Acaulospra scrobiculata Trappe & $\mathrm{x}$ & $\mathrm{x}$ & $\mathrm{x}$ & $\mathrm{x}$ \\
\hline Acaulospora sp. 1 & & & & $\mathrm{x}$ \\
\hline Archaeospora leptoticha (Schenck \& Smith) Morton \& Redecker & $\mathrm{x}$ & $\mathrm{x}$ & $\mathrm{x}$ & \\
\hline Entrophospora kentinensis Wu \& Liu & $\mathrm{x}$ & & & \\
\hline Gigaspora albida Schenck \& Smith & & & $\mathrm{x}$ & $\mathrm{x}$ \\
\hline Gigaspora margarita Becker \& Hall & & $\mathrm{x}$ & $\mathrm{x}$ & $\mathrm{x}$ \\
\hline Glomus etunicatum Becker \& Gerd. & $\mathrm{x}$ & $\mathrm{x}$ & $\mathrm{x}$ & $\mathrm{x}$ \\
\hline Glomus geosporum (Nicol. \& Gerd.) Walker & $\mathrm{x}$ & & & \\
\hline Glomus macrocarpum Tulasne \& Tulasne & $\mathrm{x}$ & $\mathrm{x}$ & $\mathrm{x}$ & $\mathrm{x}$ \\
\hline Glomus mosseae (Nicol. \& Gerd.) Gerd. \& Trappe & $\mathrm{x}$ & $\mathrm{x}$ & $\mathrm{x}$ & $\mathrm{x}$ \\
\hline Glomus spurcum Pfeiffer, Walker \& Bloss & $\mathrm{x}$ & & & \\
\hline Glomus sinuosum (Gerd. \& Bakshi) Almeida \& Schenck & $\mathrm{x}$ & & & \\
\hline Glomus sp. 1 & & & $\mathrm{x}$ & \\
\hline Glomus sp. 2 & & $\mathrm{x}$ & & \\
\hline Paraglomus occultum (Walker) Morton \& Redecker & $\mathrm{x}$ & $\mathrm{x}$ & & \\
\hline Scutellospora heterogama (Nicol. \& Gerd.) Walker \& Sanders & $\mathrm{x}$ & $\mathrm{x}$ & $\mathrm{x}$ & $\mathrm{x}$ \\
\hline Scutellospora pellucida (Nicol. \& Schenck) Walker \& Sanders & & & $\mathrm{x}$ & $\mathrm{x}$ \\
\hline Scutellospora weresubiae Koske \& Walker & $\mathrm{x}$ & & & \\
\hline Scutellospora sp. 1 & & & & $\mathrm{x}$ \\
\hline Total & 15 & 13 & 11 & 12 \\
\hline
\end{tabular}


Tabela 3. Plantas coletadas nos municípios de Piranhas e Olho d'Água do Casado nos períodos seco (PS) e chuvoso (PC), com respectivos percentuais de colonização e número de esporos de FMA na rizosfera.

Table 3. Plants collected at the municipalities of Piranhas and Olho d'Água do Casado, state of Alagoas in the dry (PS) and wet (PC) seasons, with their colonization percentage and number of FMA spores in the rhizosphere.

\begin{tabular}{|c|c|c|c|c|c|c|c|c|c|}
\hline \multirow[t]{3}{*}{ Famílias/Espécies } & \multirow[t]{3}{*}{ Nome vulgar } & \multicolumn{4}{|c|}{ Colonização \% } & \multicolumn{4}{|c|}{ Esporos/g de solo } \\
\hline & & \multicolumn{2}{|c|}{ Piranhas } & \multicolumn{2}{|c|}{ Olho d'Água } & \multicolumn{2}{|c|}{ Piranhas } & \multicolumn{2}{|c|}{ Olho d'Água } \\
\hline & & PS & $\mathrm{PC}$ & PS & $\mathrm{PC}$ & PS & $\mathrm{PC}$ & PS & $\mathrm{PC}$ \\
\hline \multicolumn{10}{|l|}{ ANACARDIACEAE } \\
\hline Spondias tuberosa Arruda & Umbuzeiro & - & - & 30,6 & 18,0 & - & - & 1,28 & 0,82 \\
\hline \multicolumn{10}{|l|}{ APOCYNACEAE } \\
\hline Aspidosperma pyrifolium Mart. & Pereiro & 9,2 & 18,6 & - & - & 2,22 & 0,84 & - & - \\
\hline A. pyrifolium & Pereiro & - & 16,6 & - & - & - & 0,84 & - & - \\
\hline A. pyrifolium & Pereiro & 7,2 & 13,1 & - & - & 3,06 & 1,52 & - & - \\
\hline \multicolumn{10}{|l|}{ BOMBACACEAE } \\
\hline Ceiba glaziovii (O. Kuntze) K. Schum. & Imbira & 10,4 & 7,6 & - & - & 8,58 & 4,38 & - & - \\
\hline \multicolumn{10}{|l|}{ BORAGINACEAE } \\
\hline Heliotropium angiospermum Murr. & Crista de galo & - & - & - & 21,0 & - & - & - & 0,50 \\
\hline H. angiospermum & Crista de galo & 31,0 & - & - & - & 2,32 & - & - & - \\
\hline H. angiospermum & Crista de galo & 13,0 & - & - & - & 0,80 & - & - & - \\
\hline \multicolumn{10}{|l|}{ BROMELIACEAE } \\
\hline $\begin{array}{l}\text { Bromelia laciniosa Mart. } \\
\text { ex Schultes f. }\end{array}$ & Macambira & - & - & 13,7 & 20,0 & - & - & 2,24 & 1,82 \\
\hline B. laciniosa & Macambira & - & - & 19,3 & - & - & - & 0,90 & - \\
\hline B. laciniosa & Macambira & - & - & 38,0 & 23,9 & - & - & 5,54 & 4,04 \\
\hline B. laciniosa & Macambira & 51,3 & 45,6 & - & - & 6,38 & 1,46 & - & - \\
\hline $\begin{array}{c}\text { Encholirium spectabile } \\
\text { Mart. ex Schultes f. }\end{array}$ & $\begin{array}{l}\text { Macambira } \\
\text { de flecha }\end{array}$ & 44,3 & 46,3 & - & - & 0,80 & 0,88 & - & - \\
\hline \multicolumn{10}{|l|}{ CACTACEAE } \\
\hline Opuntia inamoena K. Schum. & Quipá & 20,0 & 5,3 & - & - & 2,66 & 1,02 & - & - \\
\hline Pilosocereus gounellei Weber & Xique-xique & 5,6 & 9,8 & - & - & 5,54 & 3,10 & - & - \\
\hline Pilosocereus sp. & Faxeiro & - & - & 0,0 & 0,0 & - & - & 0,34 & 0,42 \\
\hline Pilosocereus sp. & Faxeiro & - & 0,0 & - & - & - & 0,84 & - & - \\
\hline Pilosocereus sp. & Faxeiro & 0,0 & - & - & - & 2,56 & - & - & - \\
\hline \multicolumn{10}{|l|}{ COMPOSTAE } \\
\hline Bidens pilosa $\mathrm{L}$. & Carrapicho & - & - & 5,6 & - & - & - & 1,98 & - \\
\hline \multicolumn{10}{|l|}{ EUPHORBIACEAE } \\
\hline Cnidoscolus obtusifolius Pohl & Favela & - & - & 10,3 & 0,0 & - & - & 3,32 & 4,00 \\
\hline Croton rhamnifolius (Baill.) Müll. Arg. & Velame & 20,6 & 12,6 & - & - & 2,40 & 1,60 & - & - \\
\hline Croton sp. & Marmeleiro & - & - & 8,1 & 14,7 & - & - & 1,16 & 0,96 \\
\hline $\begin{array}{l}\text { Ditaxis desertorum (Müll. Arg.) } \\
\text { Pax \& Hoff. }\end{array}$ & Vara branca & - & - & 9,0 & 7,0 & - & - & 2,06 & 2,48 \\
\hline Jatropha mollissima (Pohl) Baill. & Pinhão bravo & 0,0 & 37,6 & - & - & 6,48 & 1,66 & - & - \\
\hline J. ribifolia (Pohl) Baill. & Pinhão branco & - & - & 16,1 & 48,3 & - & - & 1,04 & 0,84 \\
\hline J. ribifolia & Pinhão branco & 25,6 & - & - & - & 4,06 & - & - & - \\
\hline Sapium glandulosum (L.) Morong & Burra leiteira & - & - & - & 47,0 & - & - & - & 1,94 \\
\hline S. glandulosum & Burra leiteira & 80,4 & - & - & - & 2,34 & - & - & - \\
\hline \multicolumn{10}{|l|}{ LAMIACEAE } \\
\hline Ocimum campechianum Mill. & Pissarra & - & - & 5,5 & 20,6 & - & - & 2,04 & 0,98 \\
\hline \multicolumn{10}{|l|}{ LEGUMINOSAE CAESALPINOIDEAE } \\
\hline Bauhinia cheilantha (Bong.) Steud. & Mororó & - & - & 14,9 & - & - & - & 0,40 & - \\
\hline
\end{tabular}


(cont.)

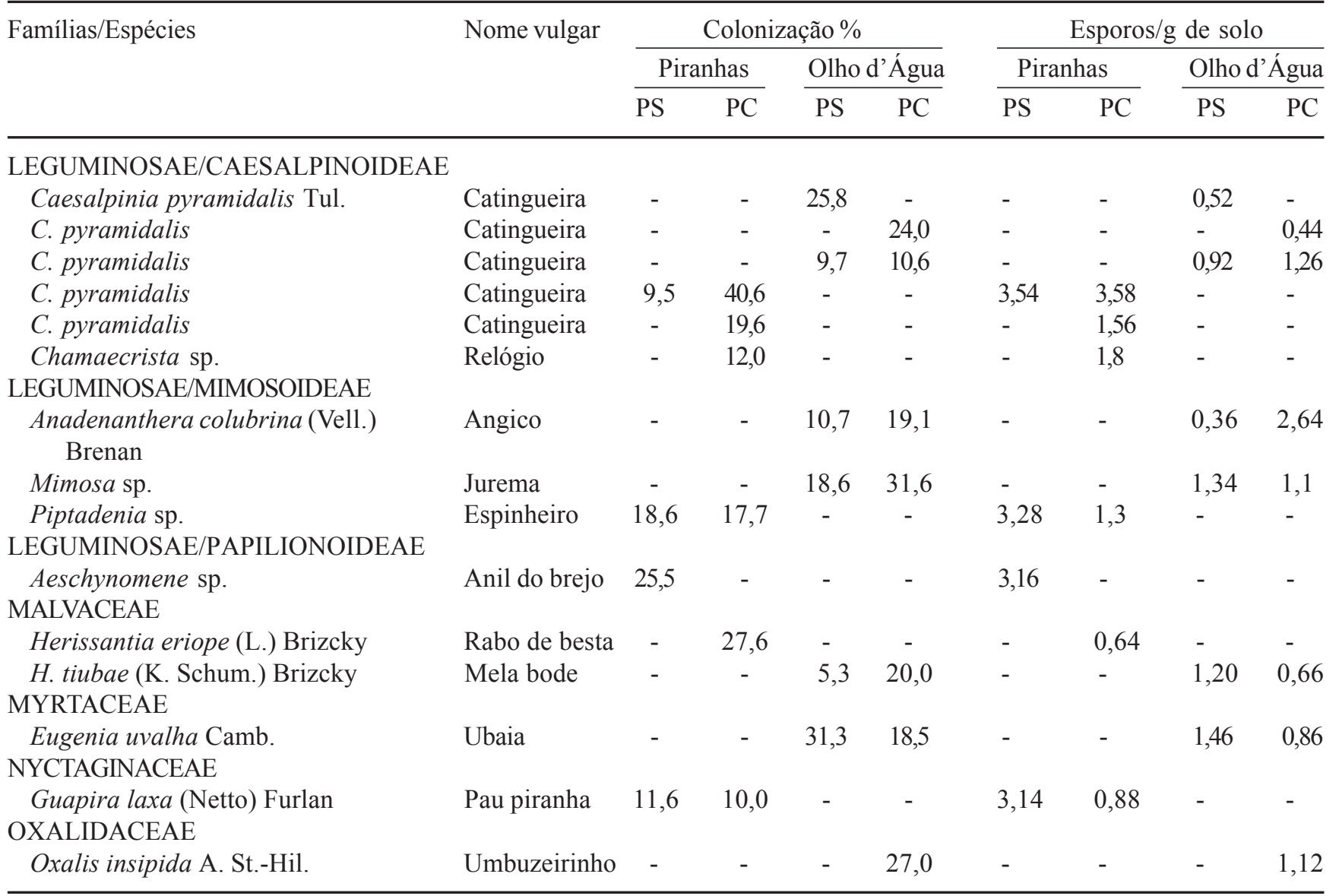

d'Água do Casado a densidade de esporos foi menor do que em Piranhas, enquanto o NMP de propágulos foi maior e se observou relação inversa entre densidade de esporos e número de propágulos infectivos (tabelas 4, 5). A média do NMP de propágulos foi significativamente diferente entre as duas áreas estudadas, sendo que esse número também diferiu em Olho d'Água do Casado, sendo maior no período seco.

A coleta permitiu avaliação da rizosfera de representantes de 14 famílias, com maior ocorrência de Leguminosae (sete gêneros e sete espécies) e Euphorbiaceae (cinco gêneros e sete espécies) (tabela 3). Das 30 espécies registradas, apenas cinco (Caesalpinia pyramidalis, Bromelia laciniosa, Jatropha ribifolia, Sapium glandulosum e Heliotropium angiospermum) foram comuns às duas áreas. $\mathrm{O}$ percentual de colonização micorrízica das plantas variou de $5 \%$ a $80 \%$ e, dos 71 espécimes examinados, apenas oito apresentaram colonização superior a $40 \%$. Não se observou formação de micorriza em Pilosocereus sp., nos dois períodos de coleta. De modo geral, os percentuais médios de colonização foram semelhantes nos dois períodos

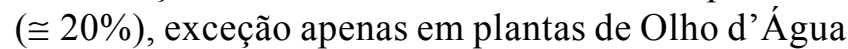
do Casado, que apresentaram menor colonização

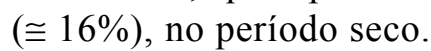

Poucos esporos de Acaulospora sp.1 foram isolados do solo e apenas no $3^{\circ}$ ciclo de multiplicação. Estes eram de coloração castanho-amarelada e subglobosos $(84-118 \times 64-90 \mu \mathrm{m})$. O gênero foi determinado pela presença de cicatriz e sáculo esporífero hialino. Esporos de Scutellospora sp. 1. (307 e $326 \mu \mathrm{m}$ ) foram isolados apenas no $2^{\circ}$ ciclo de multiplicação; o gênero foi determinado pela presença de dois grupos de parede: o externo de coloração amarelo-esverdeada e o interno hialino, com $13 \mu \mathrm{m}$ e 3,7 $\mu \mathrm{m}$ de espessura, respectivamente. A célula suspensoróide era de coloração castanha, não sendo observada placa germinativa. Glomus sp. 1 apresentou esporos amarelos, globosos a subglobosos (58-94 $\times$ 73-101 $\mu \mathrm{m}$ ), com hifa de sustentação hialina. Glomus sp. 2 foi caracterizado por esporos branco-leitosos, globosos a subglobosos $(116-186 \times 109-160 \mu \mathrm{m})$, hifa de sustentação hialina, semelhante em alguns aspectos 
Tabela 4. Densidade de esporos de FMA por g de solo nas áreas experimentais dos municípios de Piranhas (PA e PB) e Olho d'Água do Casado (AO e OB), Alagoas, nos períodos seco e chuvoso. Médias seguidas da mesma letra, maiúscula nas linhas, e minúsculas nas colunas, não diferem entre si pelo teste de Tukey a 5\%. Dados originais de número de esporos transformados em $\log (\mathrm{x}+1)$.

Table 4. FMA spores density in the experimental sites at the municipalities of Piranhas (PA and PB) and Olho d'Água do Casado (AO and OB), state of Alagoas, in the dry (PS) and wet (PC) seasons. Means followed by the same letter (capitals in lines, small in columns) do not differ from each other using Tukey test at $5 \%$.

\begin{tabular}{lcccccc}
\hline Períodos & PA & PB & Média & OA & OB & Média \\
\hline Seco & $3,76 \mathrm{Aa}$ & $3,27 \mathrm{Aa}$ & 3,51 & $1,41 \mathrm{Bb}$ & $1,70 \mathrm{Bb}$ & 1,55 \\
Chuvoso & $1,58 \mathrm{Bb}$ & $1,82 \mathrm{Bb}$ & 1,70 & $1,58 \mathrm{Bb}$ & $1,40 \mathrm{Bb}$ & 1,49 \\
Média geral & & & 2,61 & & & 1,52 \\
\hline
\end{tabular}

a Glomus albidum Walker \& Rhodes. Essas espécies não puderam ser identificadas pela escassez de esporos; a presença está sendo registrada para melhor caracterizar a diversidade de FMA nas áreas estudadas.

\section{Discussão}

Das 24 espécies de FMA identificadas, apenas seis (Glomus mosseae, G. etunicatum, G. occultum, G. spurcum, G. macrocarpum e Gigaspora albida) têm ocorrência registrada em regiões áridas da África (Diem et al. 1981; Diallo et al. 1999; Stutz et al. 2000) e da América do Norte (Stutz et al. 2000). Em relação à ocorrência no semi-árido brasileiro, foram citados anteriormente: Glomus mosseae, G. etunicatum, G. occultum, G. macrocarpum, Gigaspora margarita, Acaulospora scrobiculata e A. leptoticha em área de caatinga, na Bahia (Silva 2000), Acaulospora excavata, A. scrobiculata, A. leptoticha, A. longula, A. rehmii, Glomus etunicatum, G. macrocarpum, G. mosseae, G. occultum, G. sinuosum, Gigaspora albida, G. margarita, Scutellospora heterogama e S. pellucida em perímetro irrigado no vale do submédio São Francisco, em Pernambuco (Yano-Melo 2002) e Glomus mosseae, Acaulospora scrobiculata e A. leptoticha em agrossistemas no sertão de Pernambuco (Maia \& Trufem 1990). Não se encontrou registro de Acaulospora denticulata e Glomus spurcum em levantamentos da diversidade de FMA no Brasil.

A dificuldade para se estabelecer um padrão de distribuição dos FMA pode estar associada aos diversos fatores bióticos e abióticos relacionados aos ambientes, como também às diferentes estratégias de sobrevivência destes fungos. A distribuição das espécies de Acaulosporaceae e Glomaceae mostrou que a maioria ocorreu em solos com baixo $\mathrm{pH}$ e/ou baixo teor de fósforo. De acordo com Stürmer (1999), espécies de Acaulospora são mais freqüentemente encontradas em solos ácidos ( $\mathrm{pH}<6,2)$. Trufem $(1990,1995)$ encontrou 12 e 13 espécies deste gênero em solos com pH entre 3,5 a 5,8 e sugeriu que essas espécies são mais adaptadas a tais condições, o que indica que os níveis de $\mathrm{pH}$ no solo das áreas estudadas favorecem a ocorrência de Acaulospora. Reforçando este fato, Gomes \& Trufem (1998) encontraram 10 espécies de Acaulospora e seis de Glomus, num total de 21 espécies de FMA, associadas a solos ácidos ( $\mathrm{pH} 3,2$ e 3,4), na Ilha dos Eucaliptos (São Paulo), confirmando a presença de Acaulospora e Glomus em solos com baixo pH. Siqueira (1994) menciona que as espécies de Gigasporaceae são mais comuns em solos ácidos. No entanto, Gigaspora foi registrada em solos alcalinos (Melo et al. 1997). Em dunas, Trufem et al. (1989) encontraram maior número de espécies de Acaulospora, Gigaspora e Scutellospora, enquanto Stürmer \& Bellei (1993) referiram a predominância de $A$. scrobiculata, G. albida e G. etunicatum. Koske (1987) mencionou a ocorrência de $S$. weresubiae, em dunas da Costa Atlântica dos Estados Unidos, sugerindo que a temperatura pode atuar como regulador de distribuição desta espécie.

Allen et al. (1998) e Wilson et al. (1992) registraram maior ocorrência de espécies de Glomus e Acaulospora, respectivamente em floresta tropical decídua, no México, e em plantações de Terminalia spp., numa floresta tropical, na Costa do Marfim. As temperaturas médias anuais $\left(26\right.$ a $\left.27^{\circ} \mathrm{C}\right)$ nestes locais são semelhantes às da região semi-árida do Nordeste, sugerindo que um gradiente latitudinal de temperatura, associado a fatores edáficos, como baixo $\mathrm{pH}$, podem ter influenciado a maior ocorrência de espécies de Glomus e Acaulospora.

$\mathrm{O}$ registro de maior diversidade de FMA 
(19 espécies) nas áreas de Piranhas pode estar relacionado a dois fatores: o baixo nível de fósforo, e a maior esporulação, que favoreceu a identificação dessas espécies. No município de Olho d'Água do Casado houve menos esporulação e diversidade de FMA (14 espécies) o que pode estar relacionado, sobretudo com a maior concentração de fósforo no solo (> 40 mg.dm ${ }^{-3}$ ).

A diversidade de FMA em regiões áridas pode ser limitada, mesmo quando se utiliza cultura armadilha para melhor detectar a riqueza de espécies (Stutz et al. 2000). No entanto, em relação a outros trabalhos em ambientes áridos (Rose 1980, Diem et al. 1981, Bethlenfalvay et al. 1983, Diallo et al. 1999), a riqueza de espécies de FMA foi relativamente alta, sendo semelhante ao referido por Silva et al. (2001a), Yano-Melo (2002) e Maia \& Gibertoni (2002) também em área de caatinga. É possível que a riqueza de FMA seja maior que a encontrada, considerando-se que a multiplicação de esporos em potes de cultura, embora ajude a recuperar alguns fungos, não possibilita a identificação de todas as espécies, pois a taxa de esporulação depende, além de outros fatores, da planta hospedeira (Bever et al. 1996). Além do mais, a produção apenas eventual de esporos por alguns FMA e a presença de esporos inviáveis, dificultam a identificação e melhor descrição das espécies encontradas.

A densidade de FMA na rizosfera está relacionada com a forma agregada como os esporos são encontrados no solo e em função da distribuição, morfologia e idade fisiológica das raízes (Anderson et al. 1983), assim como depende de outros fatores que influenciam a esporulação: pluviometria, temperatura, período de insolação e espécies de FMA (Maia \& Trufem 1990, Brundrett et al. 1996). Para os diferentes sistemas, a densidade é tida como alta ou baixa em função das necessidades dos hospedeiros. Sieverding (1991) considerou como nível crítico de deficiência de propágulos de FMA no solo, aquele abaixo do qual não há resposta do hospedeiro; assim, esse nível varia de acordo com a espécie de fungo e o grau de dependência da planta à micorrização. $\mathrm{O}$ autor encontrou que o nível desejável para inoculação de plantas de mandioca com Glomus manihotis Howeler, Sieverding \& Schenck era de 12 a 36 esporos. $100 \mathrm{~g}^{-1}$ de solo, enquanto para utilizar Acaulospora appendicula Spain, Sieverding \& Schenck eram necessários entre 50 e 80 esporos. $100 \mathrm{~g}^{-1}$ de solo para obtenção da mesma resposta. É difícil separar os fatores mais importantes nessa interação, embora esteja definido que, em campo, as concentrações de propágulos de FMA são correlacionadas principalmente com a cobertura vegetal e as condições do solo (Sieverding 1991).

Em área de caatinga, Silva et al. (2001a) encontraram densidade média $<2$ esporos. g $^{-1}$ de solo, sem variação entre período seco e chuvoso. A baixa densidade de esporos pode ser comum em algumas regiões áridas, devido à presença de espécies de FMA não esporulantes (Bashan et al. 2000); talvez seja esta a razão da baixa densidade encontrada também nos solos amostrados.

As hifas de algumas espécies de FMA podem permanecer infectivas em solos secos se a esporulação não for iniciada, sugerindo uma interação entre a infectividade da hifa e o momento da esporulação (Jasper et al. 1993). Sieverding (1991) menciona que os menores números de propágulos de FMA são encontrados em áreas com vegetação degradada, e que em áreas de savana a densidade de própagulos está relacionada com a textura do solo, com os mais arenosos apresentando menores densidades, em virtude das plantas, nestes solos, sofrerem mais os efeitos da seca prolongada. Os solos das duas áreas estudadas foram predominantemente arenosos, tendo o de Olho d'Água do Casado percentagem média de $75 \%$ de areia e apenas $11 \%$ de argila.

O número de propágulos pode ser maior ou menor que o de esporos, dependendo das espécies de FMA (An et al. 1990). Menor densidade de esporos e maior número de propágulos infectivos observados nas subáreas de Olho d'Água do Casado, podem estar relacionados com a existência de diferentes mecanismos de sobrevivência dos FMA. Esta hipótese procura explicar porque algumas espécies de FMA não esporulam da mesma forma em condições ambientais semelhantes (McGee 1989). Avaliando a dinâmica sucessional, Hart et al. (2001) sugeriram que os FMA utilizam duas estratégias (colonização e persistência) para sobreviver num ambiente. No primeiro caso se enquadram as espécies mais hábeis em colonizar novos hospedeiros e no segundo, as que conseguem permanecer no sistema (solo / raiz) mesmo sob condições adversas.

De acordo com Smith \& Read (1997), maior produção de esporos pode ocorrer na fase de crescimento final do hospedeiro e no período de floração. É possível que algumas espécies de FMA que não produzam esporos com longo período de dormência germinem e, não encontrando hospedeiro disponível, acabem morrendo. Desse modo, o potencial de infectividade dos seus propágulos fica reduzido. 
A habilidade dos FMA em persistir no solo e colonizar as raízes depende, em parte, do tipo de propágulos formados (Powell \& Bagyaraj 1984) e, sendo o número de esporos baixo, como constatado em Olho d'Água do Casado ( $<2$ esporos.g ${ }^{-1}$ de solo), as hifas e/ou fragmentos de raízes colonizadas podem responder por maior potencial de infectividade (Brundrett \& Abbott 1994). É possível sugerir, portanto, que esses tipos de inóculo tenham contribuído para maior obtenção de propágulos infectivos nessa área (tabela 5).

A variação encontrada no número mais provável de propágulos de FMA também pode estar relacionada com as características físico-químicas dos solos. Rathore \& Singh (1995) observaram correlação positiva entre o número de propágulos infectivos e o nível de fósforo em diferentes tipos de solos. Tanto em Piranhas, com baixo nível de P, quanto em Olho d'Água do Casado, com teor de $\mathrm{P}$ quase dez vezes maior (tabela 1 ), houve correlação significativa $\left(\mathrm{r}^{2}=0,60, \mathrm{P}=0,02\right)$ entre o nível de fósforo no solo e o número de propágulos infectivos. O mesmo não ocorreu em relação ao $\mathrm{pH}$. Em área de caatinga nativa, Silva et al. (2001a) não obtiveram mais do que dois propágulos de FMA. $\mathrm{g}^{-1}$ de solo, com pH 6,2 e elevado nível de fósforo (141 mg.dm ${ }^{-3}$ ), confirmando que este elemento, quando em níveis elevados, pode inibir a interação planta $\times$ fungo. Johnson et al. (1991) observaram correlação negativa entre o fósforo e a infectividade de propágulos, mencionando que esta correlação se torna insignificante com $\mathrm{pH}$ constante e sugerindo que a interação $\mathrm{pH}$ e fósforo do solo pode favorecer a infectividade dos propágulos.

Abbott \& Robson (1991) sugerem que o aumento do nível de fósforo no solo pode influenciar espécies de FMA hábeis a colonizar as raízes das plantas e que a técnica do NMP pode ativar alguns propágulos que nas condições de campo não seriam infectivos. Estas hipóteses também podem justificar os diferentes números de propágulos encontrados nas áreas.

A ocorrência de plantas não micorrizadas em ecossistemas naturais de regiões semi-áridas constitui exceção, sendo a formação da simbiose parte da estratégia de tolerância das plantas aos estresses ambientais (Miller 1979). Confirmando essa observação, mais de $95 \%$ dos espécimes examinados, que constituem representantes significativos da flora local, apresentavam associação com FMA. Por outro lado, não se constatou relação entre o número de esporos na rizosfera e o percentual de colonização das plantas. Em áreas semi-áridas naturais, Reeves et al. (1979) encontraram mais de $90 \%$ das plantas micorrizadas. Em áreas de caatinga no Estado de Pernambuco, entre nove espécies de monocotiledôneas coletadas, seis apresentaram percentuais de colonização variando de $22 \%$ a $50 \%$ (Silva et al. 2001b). Por outro lado, em outras oito espécies de monocotiledôneas coletadas também em área de caatinga, no mesmo Estado, Santos et al. (2000) encontram apenas dois espécimes associados a FMA.

O grau de dependência micorrízica de plantas de caatinga pode estar relacionado ao micotrofismo facultativo das plantas de regiões áridas e semi-áridas (Allen et al. 1995). Além desse, outros fatores tais como fisiologia da raiz, susceptibilidade e competitividade nutricional das plantas hospedeiras e diferentes mecanismos de sobrevivência dos FMA interferem na colonização micorrízica. Entre as plantas examinadas, apenas Pilosocereus sp. não apresentou colonização micorrízica. Segundo Bashan et al. (2000), plântulas de cactos desenvolvem muito lentamente suas raízes até que essas possam ser colonizadas por FMA. No entanto, espécies de Cactaceae altamente colonizadas por FMA foram encontradas em região desértica da Califórnia (Bethlenfalvay et al. 1983). Foi observado que os nove indivíduos de Bromeliaceae examinados

Tabela 5. Número Mais Provável de propágulos infectivos de FMA por g de solo, nas subáreas experimentais dos municípios de Piranhas (PA e PB) e Olho d'Água do Casado (OA e OB), Alagoas, nos períodos seco e chuvoso. Dados originais do NMP. Para estatística, dados transformados em $\log (\mathrm{x})$.

Table 5. Most probable number of FMA infective propagules per $\mathrm{g}$ of soil, in the experimental sub-areas at the municipalities of Piranhas (PA and PB) and Olho d'Água do Casado (AO and OB), state of Alagoas, in the dry and wet seasons. NMP original data. Data transformed for $\log (\mathrm{x})$.

\begin{tabular}{lccccrr}
\hline Períodos & PA & PB & Média & OA & OB & Média \\
\hline Seco & 0,82 & 1,88 & $1,35 \mathrm{a}$ & 10,0 & 13,18 & $11,60 \mathrm{~b}$ \\
Chuvoso & 1,40 & 2,39 & $1,90 \mathrm{a}$ & 3,44 & 5,97 & $4,70 \mathrm{a}$ \\
Média geral & & & $1,62 \mathrm{a}$ & & & $8,15 \mathrm{~b}$ \\
\hline
\end{tabular}


apresentavam colonização (de 13\% a 51\%). Formação de associação micorrízica em plantas desta família, em ambientes naturais, havia sido referida apenas por Santos et al. (2000), em espécimen coletado no estado de Pernambuco. Confirma-se a importância dos FMA no estabelecimento das plantas e na manutenção de ecossistemas semi-áridos.

Agradecimentos - À equipe do Programa de Biodiversidade - Projeto Xingó/UFPE/UFRPE/CHESF pelo apoio na escolha das áreas e nas coletas. Aos colegas Joana Angélica C. Brandão, Nicácio de Oliveira Freitas e Bruno Tomio Goto, (bolsistas Pibic/UFPE) e à equipe do Laboratório de Micorrizas/UFPE, pela colaboração durante o desenvolvimento deste trabalho. Ao Dr. Everardo Valadares de S. B. Sampaio (UFPE), pelo auxílio na análise estatística e discussão dos resultados. L.C. Maia e R.G. Souza agradecem as bolsas concedidas, respectivamente pelo CNPq (Pesquisa) e Capes (Mestrado). Os dados do município de Piranhas foram fornecidos pela Divisão de Gestão de Recursos Hídricos DORH-CHESF-PE e do município de Olho d'Água do Casado, referentes aos do município de Delmiro Gouveia, fornecidos pela Secretaria de Estado de Recursos Hídricos e Irrigação de Alagoas (SERHI).

\section{Referências bibliográficas}

ABBOTT, L.K. \& ROBSON, A.D. 1991. Factors influencing the occurrence of vesicular-arbuscula mycorrhizae. Agriculture, Ecosystems and Environment 35:121-150.

ALLEN, E.B., ALLEN, M.F., HELM D.J., TRAPPE, J.M., MOLINA, R. \& RINCON, E. 1995. Patterns and regulation of mycorrhizal plant and fungal diversity. Plant and Soil 170:47-62.

ALLEN, E.B., RINCON, E., ALLEN, M.F., JIMENEZ, A.P. \& HUANTE, P. 1998. Disturbance and seasonal dynamics of mycorrhizae in a tropical deciduous forest in México. Biotropica 30:61-274.

AN, Z.Q., HENDRIX, J.W., HERSHMAN, D.E. \& HENSON, G.T. 1990. Evaluation of the "most probable number" (MPN) and wet-sieving methods for determining soilborne populations of endogonaceous mycorrhizal fungi. Mycologia 85:576-581.

ANDERSON, R.C., LIBERTA, A.E., DICKMAN, L.A. \& KATZ, A.J. 1983. Spatial variation in vesicular-arbuscular mycorrhiza spore density. Bulletin of the Torrey Botanical Club 110:519-525.

AUGÉ, R.M., STODOLA, A.J.W., TIMS, J.E. \& SAXTON, A.M. 2001. Moisture retention properties of a mycorrhizal soil. Plant and Soil 230:87-97.

BASHAN, Y., DAVIS, E.A., CARRILLO-GARCIA, A. \& LINDERMAN, R.G. 2000. Assessment of mycorrhizal inoculum potencial in relation to the establishment of cactus seedlings under mesquite nurse-trees in the Sonoran Desert. Applied Soil Ecology 14:165-175.
BETHLENFALVAY, G.J., DAKESSIAN, S. \& PACOVSKY, R.S. 1983. Mycorrhizae in a southern California desert: ecological implications. Canadian Journal of Botany 62:519-524.

BEVER, J.D., MORTON, J.B., ANTONOVICS, J. \& SCHULTZ, P.A. 1996. Host-dependent sporulation and species diversity of arbuscular mycorrhizal fungi in a mown grassland. Journal of Ecology 84:71-82.

BROWER, J.E. \& ZAR, J.H. 1984. Community similarity. In Field \& Laboratory for General Ecology. (J.E. Brower \& J.H. Zar, eds.). Win C. Brown Publishers, Dubuque, p.161-164.

BRUNDRETT, M.C. \& ABBOTT, L.K. 1994. Mycorrhizal fungus propagules in the Jarrah forest. I. Seasonal study of inoculum levels. New Phytologist 127:539-546.

BRUNDRETT, M.C., ASHWATH, N. \& JASPER, D.A. 1996. Mycorrhizas in the Kakadu region of tropical Australia. Plant and Soil 184:173-184.

DIALLO, A.T., SAMB, P.I. \& DUCOUSSO M. 1999. Arbuscular mycorrhizal fungi in the semi-árida areas of Senegal. European Journal of Soil Biology 35:65-75.

DIEM, H.G., GUEYE, I., GIANINAZZI-PEARSON, V., FORTIN, J.A. \& DOMMERGUES, Y.R. 1981. Ecology of VA mycorrhizae in the tropics: the semiarid zone of Senegal. Acta Ecologica/Ecology Plantarum 2:53-62.

DRUMOND, M.A., KIILL, L.H.P., LIMA, P.C.F., OLIVEIRA, M.C., OLIVEIRA, V.R., ALBUQUERQUE, S.G., NASCIMENTO, C.E.S. \& CAVALCANTE, J. 2000. Estratégias para o uso sustentável da biodiverdidade da caatinga. In Seminário para avaliação e identificação de ações prioritárias para a conservação, utilização sustentável e repartição de benefícios da biodiversidade do bioma Caatinga. Embrapa/Cpatsa, UFPE e Conservation International do Brasil, Petrolina.

FISHER, R.A. \& YATES, F. 1970. Statistical tables for biological, agricultural and medical research. Hafner Publ. Comp., Davien.

GERDEMANN, J.W. \& NICOLSON, T.H. 1963. Spores of mycorrhizal Endogone species extracted from soil by wet sieving and decanting. Transactions of the British Mycological Society 46:235-244.

GIOVANNETTI, M. \& MOSSE, B. 1980. An evaluation of techniques for measuring vesicular arbuscular mycorrhizal infection in roots. New Phytologist 84:489-500.

GOMES, S.P. \& TRUFEM, S.F.B. 1998. Fungos micorrízicos arbusculares (Glomales, Zygomycota) na Ilha dos Eucaliptos, Represa do Guarapiranga, São Paulo, SP. Acta Botanica Brasilica 12:395-401.

HART, M.M., READER, R.J. \& KLIRONOMOS, J.N. 2001. Life-history strategies of arbuscular mycorrhizal fungi in relation to their sucessional dynamics. Mycologia 93:1186-1194. 
JACOMINE, P.K.T., CAVALCANTI, A.C., PESSOA, S.C.P. \& SILVEIRA, C.O. 1975. Levantamento exploratório Reconhecimento de solos do Estado de Alagoas. Centro de Pesquisa Pedológica - Embrapa e Divisão de Recursos Renováveis - Sudene, Boletim Técnico 35, Maceió.

JASPER, D.A., ABBOTT, L.K. \& ROBSON, A.D. 1993. The survival of infective hyphae of vesicular-arbuscular mycorrhizal fungi in dry soil: an interaction with sporulation. New Phytologist 124:473-479.

JENKINS, W.R. 1964. A rapid centrifugal-flotation technique for separating nematodes from soil. Plant Disease Report 48:692.

JOHNSON, N.C., ZAK, D.R., TILMAN, D. \& PFLEGER, F.L. 1991. Dynamics of vesicular-arbuscular mycorrhizae during old field sucession. Oecologia 80:349-358.

KOSKE, R.E. 1987. Distribution of VA mycorrhizal fungi along a latitudinal temperature gradient. Mycologia 79:55-68.

MAIA, L.C. \& GIBERTONI, T.B. 2002. Fungos registrados no semi-árido nordestino. In Vegetação e Flora da Caatinga. (E.V.S.B. Sampaio, A.M. Giulietti, J. Virgínio \& C.F.L. Gamarra-Rojas, eds.). Associação Plantas do Nordeste e Centro Nordestino de Informações sobre Plantas, Recife, p.163-176.

MAIA, L.C. \& TRUFEM, S.F.B. 1990. Fungos micorrízicos vesículo-arbusculares em solos cultivados no Estado de Pernambuco, Brasil. Revista Brasileira de Botânica 13:89-95.

MCGEE, P.A. 1989. Variation in propagule numbers of vesicular-arbuscular mycorrhizal fungi in a semi-arid soil. Mycological Research 92:28-33.

MELO, A.M.Y., MAIA, L.C. \& MORGADO, L.B. 1997. Fungos micorrízicos arbusculares em bananeiras cultivadas no vale do submédio São Francisco. Acta Botanica Brasilica 11:115-121.

MILLER, R.M. 1979. Some occurrences of vesiculararbuscular mycorrhiza in natural and disturbed ecosystems of the Red Desert. Canadian Journal of Botany 57:619-623.

MILLER, R.M. \& KLING, M. 2000. The importance of integration and scale in the arbuscular mycorrhizal symbiosis. Plant and Soil 226:295-309.

MORTON, J.B. 1993. Problems and solutions for the integration of glomalean taxonomy, systematic biology, and the study of endomycorrhizal phenomena. Mycorrhiza 2:97-109.

PHILLIPS, J.M. \& HAYMAN, D.S. 1970. Improved procedures for clearing roots and staining parasitic and vesicular mycorrhizal fungi for rapid assessment of infection. Transactions of the British Mycological Society 55:158-161.

POWELL, C.L. \& BAGYARAJ, J.D. 1984. Fungal Species. In VA Mycorrhiza. (C.L. Powell \& J.D. Bagyaraj, eds.). CRC Press, Boca Raton, p.43-50.
RATHORE, V.P. \& SINGH, H.P. 1995. Quantification and correlation of vesicular-arbuscular mycorrhizal propagules with soil properties of some mollisols of Northern India. Mycorrhiza 5:201-203.

REEVES, F.B., WAGNER, D., MOORMAN, T. \& KIEL, J. 1979. The role of endomycorrhizae in revegetation practices in the semi-arid West. I. A comparison of incidence of mycorrhizae in severely disturbed vs. natural environments. American Journal of Botany 66:6-13.

ROSE, S.L. 1980. Vesicular-arbuscular endomycorrhizal associations of some desert plants of Baja California. Canadian Journal of Botany 59:1056-1060.

SANTOS, B.A., SILVA, G.A., MAIA, L.C. \& ALVES, M.V. 2000. Mycorrhizae in Monocotyledonae of Northeast Brazil: subclasses Alismatidae, Arecidae and Zingiberidae. Mycorrhiza 10:151-153.

SAMPAIO, E.V.S.B. 1995. Overview of the Brazilian caatinga. In Seasonally dry tropical forests. (S.H. Bullock, A.M. Harold \& E. Medina, eds.). Cambridge University Press, Cambridge, p.35-63.

SCHENCK, N.C. \& PÉREZ, Y. 1990. Manual for the identification of VA mycorrhizal fungi. $3^{\text {rd }} \mathrm{ed}$. Synergistic Publ., Gainesville.

SIEVERDING, E. 1991. Vesicular-arbuscular mycorrhiza management in tropical agrosystems. Deutsche Gesellschaft Technische Zusammenarbeit (GTZ) GmbH, Eschborn.

SILVA, G.A. 2000. Potencial de infectividade de fungos micorrízicos arbusculares oriundos de área de caatinga nativa e degradada por mineração. Dissertação de mestrado, Universidade Federal de Pernambuco, Recife.

SILVA, G.A., MAIA, L.C., SILVA, F.S.B. \& LIMA, P.C.F. 2001 a. Potencial de infectividade de fungos micorrízicos arbusculares oriundos de área de caatinga nativa e degradada por mineração, no Estado da Bahia, Brasil. Revista Brasileira de Botânica 24:135-143.

SILVA, G.A., SANTOS, B.A., ALVES, M.V. \& MAIA, L.C. 2001b. Arbuscular mycorrhiza in species of Commelinidae (Liliopsida) in the State of Pernambuco (Brazil). Acta Botanica Brasilica 15:155-165.

SIQUEIRA, J.O. 1994. Micorrizas arbusculares. In Microrganismos de importância agrícola. (R.S. Araújo \& M. Hungria, eds.). Embrapa-SPI, Brasília, p.235-249.

SKUJINS, J. \& ALLEN, M.F. 1986. Use of mycorrhizae for land rehabilitation. Mircen Journal 2:161-176.

SMITH, S.E. \& READ, D.J. 1997. The symbiontes forming VA mycorrhizas. In Mycorrhizal Symbiosis. (S.E. Smith \& D.J. Read, eds.). Academic Press, San Diego, p.33-80.

STATISTIC FOR WINDOWS. 1995. StatSoft, Tulsa.

STÜRMER, S.L. \& BELLEI, M.M. 1993. Composition and seasonal variation of spore populations of arbuscular mycorrhizal fungi in dune soils on the Island of Santa Catarina, Brazil. Canadian Journal of Botany 72:359-363. 
STÜRMER, S.L. 1999. Evolução, classificação e filogenia dos fungos micorrízicos arbusculares. In Inter-relação fertilidade, biologia do solo e nutrição de plantas. (J.O. Siqueira, F.M.S. Moreira, A.S. Lopes, L.R.G. Guilherme, V. Faquin, A.E. Furtini Neto \& J.G. Carvalho, eds.). Sociedade Brasileira de Ciência do Solo, Lavras, p.797-817.

STUTZ, J.C., COPEMAN, R., MARTIN, C.A. \& MORTON, J.B. 2000. Patterns of species composition and distribution of arbuscular mycorrhizal fungi in arid regions of Southwestern North America and Namibia, África. Canadian Journal of Botany 78:237-245.

SYLVIA, D.M. 1992. Quantification of external hyphae of vesicular-arbuscular mycorrhizal fungi. In Methods in Mycrobiology: Techniques for the Study of Mycorrhiza (J.R. Norris, D.J. Read \& A.K. Varma, eds.). Academic Press, New York, p.53-66.

TARAFDAR, J.C. \& PRAVEEN-KUMAR. 1996. The role of vesicular arbuscular fungi on crop, tree and grasses grown in an arid environment. Journal of Arid Environment 34:197-203.

TOMÉ JÚNIOR, J.B. 1997. Interpretação dos resultados. In Manual para interpretação de análise do solo. (J.B. Tomé Júnior, ed.). Agropecuária, Guaíba, p.89-107.
TRUFEM, S.B. 1990. Aspectos ecológicos de fungos micorrízicos vesículo-arbusculares da mata tropical úmida da Ilha do Cardoso, SP, Brasil. Acta Botanica Brasilica 4:31-45.

TRUFEM, S.B. 1995. Aspectos ecológicos de fungos micorrízicos arbusculares na rizosfera de plantas de restinga da Ilha do Cardoso, SP, Brasil. Revista Brasileira de Botânica 18:51-60.

TRUFEM, S.B., OTOMO, H.S. \& MALATINSZKY, S.M.M. 1989. Fungos micorrízicos vesículo-abusculares em rizosfera de plantas em dunas do Parque Estadual da Ilha do Cardoso, São Paulo, Brasil. (1) Taxonomia. Acta Botanica Brasilica 3:141-152.

WILSON, J., INGLEBY, K., MASON, P.A., IBRAHIM, K. \& LAWSON, G.J. 1992. Long-term changes in vesiculararbuscular mycorrhizal spore populations in Terminalia plantations in Côte d'Ivoire. In Mycorrhizas in Ecosystems. (D.J. Read, D.H. Lewis, A.H. Fitter \& I.J. Alexander, eds.). CAB Internacional, Cambridge, p.268-275.

YANO-MELO, A.M. 2002. Biologia de fungos micorrízicos arbusculares em solos salinizados. Tese de doutorado, Universidade Federal de Pernambuco, Recife. 